\title{
Evidence for two distinct perceptual alterations in irritable bowel syndrome
}

\author{
B D Naliboff, J Munakata, S Fullerton, R H Gracely, A Kodner, F Harraf, E A Mayer
}

CURE Digestive

Diseases Research

Center/Neuroenteric

Disease Program,

WLA VA Medical

Center, and UCLA,

Los Angeles,

CA 90073, USA

B D Naliboff

J Munakata

$S$ Fullerton

A Kodner

F Harraf

E A Mayer

Department of

Medicine

J Munakata

$S$ Fullerton

E A Mayer

Department of

Physiology

E A Mayer

Department of

Psychology

B D Nalibof

Neuropathic Pain and

Pain Assessment

Section, NAB, NIDR,

NIH, Bethesda,

MD 20892, USA

R H Gracely

Correspondence to:

Dr B D Naliboff, WLA VA

Medical Center,

Neuroenteric Biology Group,

Bldg 115/CURE, 11301

Wilshire Blvd, Los Angeles,

CA 90073, USA.

Accepted for publication 2 May 1997

\begin{abstract}
Background and aims-Visceral hyperalgesia has been implicated as a factor contributing to symptom generation in irritable bowel syndrome (IBS). However, previous studies using intestinal balloon distension have used psychophysical procedures which do not provide adequate and unbiased measures of visceral sensitivity.
\end{abstract}

Methods-Three psychophysical tasks were examined in 45 patients with IBS (positive Rome criteria) and 14 controls using rectal balloon distension with a computerised distension device. Discomfort threshold and tolerance were assessed during an ascending series of phasic pressure stimuli and during an interactive threshold tracking procedure. In addition, stimulus response functions were generated from intensity and unpleasantness ratings of the rectal distensions.

Results-Discomfort threshold and tolerance for the ascending stimuli were significantly lower for the patients with IBS compared with the controls. In contrast, discomfort thresholds during the tracking procedure and stimulus response curves for the ascending series were not different between the groups. A factor analysis of the psychophysical data was consistent with the presence of two distinct and unrelated perceptual alterations related to rectal distension: hypervigilance for visceral stimuli, manifested as lowered response criteria for using the descriptor "discomfort"; and rectal hypersensitivity, manifested as a lower discomfort threshold and left shift of the stimulus response curves. Conclusions-Patients with IBS as a group have a greater propensity to label visceral sensations negatively and show a lower tolerance for rectal balloon distension. A subgroup of patients also have baseline rectal hypersensitivity, assessed by unbiased measures of discomfort threshold and stimulus intensity judgements.

(Gut 1997; 41: 505-512)

Keywords: irritable bowel syndrome; visceral hypersensitivity; pain threshold

A variety of clinical observations including the presence of recurring abdominal pain, and excessive pain during endoscopic examinations of the sigmoid colon are consistent with a role for visceral hyperalgesia in irritable bowel syn- drome (IBS) symptom generation. Experimental evidence from studies assessing visceral sensitivity demonstrates that a variety of perceptual abnormalities in relation to gastrointestinal stimuli are more frequent in patients with IBS. ${ }^{1-3}$ For example, in a recent study, Mertz et $a l^{\beta}$ found that patients with IBS had a significantly lower median discomfort threshold for a 30 second rectal balloon stimulus compared with a normal population. If lowered threshold and two other perceptual abnormalities were considered (altered area of sensory referral and increased ratings of the rectal sensations from balloon distension), $95 \%$ of patients with IBS had at least one abnormality. Only $7 \%$ of a control population had at least one of the three sensory findings. Other studies have also found significant perceptual alterations in IBS populations including lowered discomfort thresholds for balloon distension of the small intestine, the colon, and the rectosigmoid..$^{1-5}$

Three difficulties arise with the interpretation of these studies as evidence for afferent hypersensitivity being causative in IBS symptoms of abdominal pain and/or discomfort. Firstly, a significant percentage of symptomatic patients with IBS do not show altered rectal thresholds to balloon distension under baseline conditions, although they may demonstrate an abnormal pattern of referral of visceral sensations. Secondly, the techniques used in previous studies may not yield reliable estimates of true pain or discomfort thresholds. The aversive sensation associated with rectal balloon distension at pressures used in human studies is typically well below that for true visceral pain. ${ }^{6}$ Furthermore, thresholds determined from the lowest pressure (or volume) which a subject labels as uncomfortable during an ascending series of stimuli can be greatly affected by non-sensory information, a phenomenon referred to as response bias. ${ }^{78}$ The predictable increase in stimulus magnitude during the ascending series allows for bias of subjects' judgements based on anticipation of a higher level stimulus, fear of increasing discomfort, and a tendency to label visceral sensations in aversive terms. In contrast, sensory testing techniques which involve unpredictable stimulus intensities, such as tracking or staircase procedures, lessen the chances that these nonsensory cues will significantly influence threshold ratings. ${ }^{8}$ The problem of response bias in assessments of visceral sensitivity may be especially important in light of data showing that patients with IBS who seek health care have significantly more psychological distress than 
either control subjects or persons with IBS symptoms who have not sought medical treatment. ${ }^{9}$ In addition, it has been shown recently that patients with IBS have a selective affective bias in recognition memory, implying an overall negative focus especially with regard to internal states, ${ }^{10}$ and show abnormal illness attitudes beyond that related to depression or symptom experience. ${ }^{11} \mathrm{~A}$ third methodological issue is that sensation (or alterations in sensation) at the threshold of discomfort is typically of much less clinical interest than sensation at higher intensities (moderate to severe discomfort or pain). Assessment of a discomfort threshold (even if unbiased) is therefore of significant interest to the extent that it is reliably related to suprathreshold hypersensitivity (sensitivity above discomfort or pain threshold) or to altered stimulus ratings for moderate or higher stimulus intensities (stimulus response functions).

The goal of the present study was to extend our previous investigations ${ }^{3}$ by further characterising the perceptual alterations to rectal stimuli in symptomatic patients with IBS. We compared the discomfort thresholds obtained with the traditional method of an ascending series with two established psychophysical approaches for sensory assessment: an unbiased measure of threshold from a stimulus tracking task; and stimulus response curves obtained using sensory and affective verbal descriptor scales. ${ }^{12}$ Specifically, we sought to answer the following questions: Do IBS and control groups differ in their threshold for use of common visceral sensory descriptors (including discomfort) during an ascending series of phasic pressure stimuli? Do IBS and control groups differ on rectal pressure discomfort threshold as determined by an unbiased stimulus tracking procedure? Is there a group difference in the slope or intercept of the stimulus response curves for sensory and affective ratings of visceral stimuli? Part of these results have been published in abstract form. ${ }^{13}$

\section{Methods}

SUBJECTS

The IBS patient group consisted of 45 consecutive patients seen at the UCLA Center for Functional Bowel Disorders and Abdominal Pain. Patients participated in the study protocol as part of the comprehensive anorectal manometry performed on all patients with lower gastrointestinal disorders presenting to the Center. The population of patients with IBS seen at the Center is characterised as follows: approximately $30 \%$ are self-referred, $30 \%$ are referred by a private gastroenterologist, and $30 \%$ are referred by primary care physicians. All patients met the Rome diagnostic criteria for IBS $^{14}$ and had more than three positive Manning criteria ${ }^{15}$ with no evidence of organic disease. The diagnosis of each patient was confirmed by staff gastroenterologists experienced in the diagnosis of functional bowel disorders.

Fourteen asymptomatic control subjects without evidence of an acute or chronic illness were recruited by newspaper advertisement. In particular, there was no evidence in any of the subjects of an acute or chronic pain syndrome or persistent abdominal symptoms either by bowel symptom questionnaire, personal history, or physical examination. According to subjects' reports, the majority had never participated in a research study before, and none of the subjects had ever participated in a study involving intubation of the gastrointestinal tract. All studies were approved by the Institutional Review Board of the WLA-VA Medical Center.

MATERIALS AND APPARATUS

Psychological symptom checklist

All participants completed the SCL-90 symptom checklist ${ }^{16}$ which assesses acute psychological symptom severity in the following areas: anxiety, depression, hostility, interpersonal sensitivity, obsessive compulsive behaviour, paranoia, phobic behaviour, psychosis, and somatisation.

\section{Visceral stimulation device}

The use of a computer driven volume displacement device allowed for controlled inflation of the rectal balloon to target pressure stimuli. ${ }^{35}$ The distension device was programmed to deliver distension at a rapid volume rate $(14.5 \mathrm{ml} / \mathrm{s})$ to constant pressure plateaus, simultaneously to record pressures and volumes (sampling rate $1 / \mathrm{s}$ ), and to record the responses of the subject on a hand held four button box (buttons labelled: "no sensation", "moderate sensation", "discomfort", and "pain").

A latex balloon was attached to a silastic elastomer tube (external diameter, 18F) and tied at both proximal and distal ends (MAKLA, Los Angeles, California). The distance between the attachment sites was $11 \mathrm{~cm}$. Distension to a maximal volume of $450 \mathrm{ml}$ resulted in a spherical balloon shape. Prior to, and following the procedure, each balloon was inflated repeatedly to rule out any leak, and measure intrinsic compliance. In preliminary studies, it was found that during the initial three balloon inflations, the maximally reached balloon pressure decreased by $10 \%$. Following this initial decrease, the volume pressure relationship of the balloon remained constant during subsequent inflations. The lubricated balloon was inserted into the rectum so that the distal attachment site was $4 \mathrm{~cm}$ from the anal verge. The tube was then secured with tape. In vitro and in vivo validation of this distension device have been published previously. ${ }^{17} 18$

\section{EXPERIMENTAL PROCEDURE}

Participants were placed in the left lateral decubitus position on a padded table. Although the examiner was always present, interaction with the participants ceased after the initial explanation of the respective task. Subjects had no visual or auditory cues to anticipate type, or time course of distensions nor were they instructed about the nature of the distension protocols. They were given specific instructions regarding how to rate each stimulus presentation before each task. Following a 12 hour fast and application of two Fleets enemas 
(CB Fleet Co. Inc., Lynchburg, Virginia), two distension procedures were used to evaluate rectal perception: first an ascending series and then an interactive tracking task.

\section{Ascending series}

An ascending series of 30 second rapid phasic pressure stimuli $(15,10,25,20,35,30,45,40$, $50,60 \mathrm{~mm} \mathrm{Hg}$ ) were separated by a 30 second inter-stimulus rest $(5 \mathrm{~mm} \mathrm{Hg}){ }^{3}$ At each pressure stimulus of the 10 trial sequence, the participant rated the stimulus with validated verbal descriptor rating scales of sensory and affective intensity ${ }^{12}$; and labelled the sensation using one or more words from a list of qualitative descriptors of visceral sensation (no sensation, abdominal cramps, bloating, fullness, gas, stool, urgency, discomfort). If the pressure became intolerable the procedure was stopped.

\section{Sensory tracking}

The electronic barostat was programmed to deliver a series of intermittent phasic stimuli (15 seconds duration; $5 \mathrm{~mm} \mathrm{Hg}$ increments) separated by a $5 \mathrm{~mm} \mathrm{Hg}$ interpulse rest (30 seconds duration) within an interactive stimulus tracking procedure. ${ }^{19}$ At 10 seconds into each stimulus period, subjects were prompted by the electronic barostat to report the intensity of their perceived sensations by triggering the push button marker device connected to the barostat. If the subject indicated a sensation below the tracked intensity (discomfort), the following stimulus increased by $5 \mathrm{~mm} \mathrm{Hg}$. Conversely, the following pressure step was randomised to stay the same or decrease by $5 \mathrm{~mm} \mathrm{Hg}$ if the subject triggered discomfort. The random element was placed in the tracking procedure to mask the relationship between ratings and subsequent stimulus change and therefore decrease potential scaling bias. ${ }^{20}$ In all instances, if the participant indicated onset of pain the next stimulus was decreased by $5 \mathrm{~mm} \mathrm{Hg}$. The tracking task requires subjects repeatedly to make a single judgement of a series of similar, but nonidentical and unpredictable stimuli. This task forces the subject to make judgements based on sensory information alone, and therefore favours production of a reliable and unbiased threshold.

EVALUATION OF OUTCOME PARAMETERS

The following parameters of the psychophysical tasks were examined: sensory thresholds (in $\mathrm{mm} \mathrm{Hg}$ ) for the presence of specific visceral sensations during the ascending series; stimulus response functions of sensory and affective intensity during the ascending series; rectal wall tension (computed from pressure and volume measurements) at each pressure step during the ascending series (see later); and discomfort threshold (in $\mathrm{mm} \mathrm{Hg}$ ) during the interactive stimulus tracking procedure.

\section{Ascending series sensation thresholds}

Specific visceral sensation thresholds were determined during the ascending series from the lowest pressure at which a subject used a particular descriptor. For example, if discom- fort was first checked at $30 \mathrm{~mm} \mathrm{Hg}, 30 \mathrm{~mm} \mathrm{Hg}$ would be the discomfort threshold for that subject.

\section{$S-R$ functions}

Stimulus response functions for both sensory intensity and unpleasantness of the pressure stimuli were analysed. The verbal descriptor sensory scale consists of a $20 \mathrm{~cm}$ vertical bar flanked by descriptors of increasing intensity (no sensation, faint, very weak, weak, very mild, mild, moderate, barely strong, slightly intense, strong, intense, very intense, and extremely intense). The affective scale is a similar graphical rating scale of unpleasantness descriptors (neutral, slightly unpleasant, slightly annoying, unpleasant, annoying, slightly distressing, very unpleasant, distressing, very annoying, slightly intolerable, very distressing, intolerable, very intolerable). Placement of words along each scale was determined from their relative log intensity rating in a normative study. ${ }^{12}$ The validity of these scales for assessing the perceived intensity of visceral sensations has recently been confirmed. ${ }^{21}$ Subjects were instructed to rate the "intensity of the sensation from the previous inflation" immediately after each inflation on each of the scales using the words as guides. The scales were printed on separate sheets of paper.

\section{Wall tension}

Wall tension was estimated at each distending stimulus during the semi-random ascending series for all participants. Although the precise geometry of the rectum is not known, rectal wall tension was estimated by assuming a cylinder length of $11 \mathrm{~cm}$ (balloon length). ${ }^{3} 17$ Radius at each pressure stimulus was derived from the volume of the cylinder $\left(\mathrm{V}=\mathrm{r}^{2} \mathrm{~L}\right.$, in which $\mathrm{L}=11 \mathrm{~cm})$. Using LaPlace's law $(\mathrm{T}=2 \mathrm{pr}$ ), wall tension ( $T$ ) was calculated from the estimated balloon radius and the delivered pressure (p). Two wall tension values were computed for each stimulus: the wall tension when the balloon initially reached the target pressure, and the wall tension at maximum volume for the target pressure. Rectal tone (the volume required to maintain baseline pressure) remained constant during the distension procedures.

\section{Tracking discomfort threshold}

Discomfort threshold for the stimulus tracking task was defined as the average of the last six pressures delivered or the average of all pressures delivered following the first indication of discomfort (if less than six). Length of the tracking task was 10 minutes (14 stimulus trials) which is sufficient to give stable discomfort thresholds. To validate the stability of the 10 minute task, we compared the thresholds at 10 and 15 minutes for a group of IBS and control subjects $(n=24)$ given a longer tracking period. The results indicated that the thresholds determined at 10 and 15 minutes were highly correlated $(r=0.79, \mathrm{p}<0.01)$ and there was no significant mean difference between the two thresholds. 
DATA ANALYSIS

Initial data analyses compared the IBS and control groups on responses to the psychophysical tasks. Subsequent analyses compared the various measures with each other and examined differences between hypersensitive and normosensitive patients with IBS. Group results are expressed as mean (SEM) throughout.

\section{Results}

DEMOGRAPHICS VARIABLES

Seventy six per cent of the patients with IBS and $43 \%$ of the control subjects were female $\left(\chi^{2}\right.$ non-significant). There was a significant difference in age between the IBS and control groups $(\mathrm{p}<0.05)$ with patients with IBS being older (mean 54.7 (2.9) years) than controls (mean 42.2 (4.9) years). Examination of the correlations between age and the various psychophysical variables indicated no significant relationships ( $r=0.01-0.21$, all non-significant), and addition of age as a covariate in the group comparisons reported below also did not change the pattern of differences found.

PSYCHOLOGICAL SYMPTOMS

SCL-90 scores for patients with IBS were significantly elevated compared with controls on symptom scales of somatisation, depression anxiety, phobia, and general symptom severity (all $\mathrm{p}<0.05)$ (fig 1 )

ASCENDING SERIES THRESHOLDS FOR THE

VISCERAL SENSATION DESCRIPTORS

Analysis of the pressures at which the various visceral sensation descriptors (including discomfort) were first used showed significant mean group differences for discomfort $(t=3.18$; $\mathrm{p}<0.01)$. The average pressure at which patients with IBS first used discomfort was 24.7 (2.1) $\mathrm{mm} \mathrm{Hg}$ compared with 38.0 (2.3) $\mathrm{mm} \mathrm{Hg}$ for the controls. Figure 2 shows the cumulative percentage of subjects in each group using discomfort across the pressure steps. A non-parametric analysis of these two distributions also indicated that they were significantly different (Kolmogorov-Smirnov, $\mathrm{z}=2.98$, $\mathrm{p}<0.01)$. There were no significant group

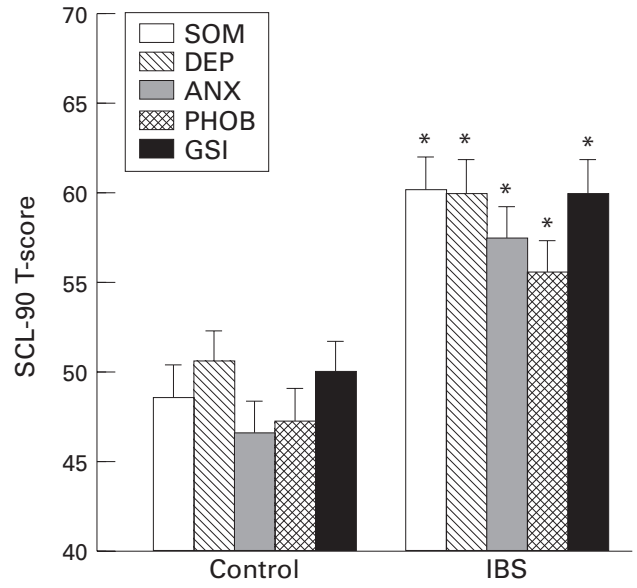

Figure 1: $\quad S C L-90$ scores for IBS and control groups. SOM, somatisation; DEP, depression; $A N X$, anxiety; PHOB, phobia; GSI, global symptom index. ${ }^{*} p<0.05$ in comparison with IBS group. differences in the average ascending thresholds for any of the other descriptors. However, the two groups do show some differences in use of the descriptors, with the controls showing a more reliable ordering of descriptor usage with increasing pressure. This is evident when the descriptors are arranged, as in fig 3, by increasing average threshold, from fullness through cramping and discomfort. The IBS group, on the other hand, does not show a similar ordering of the descriptor thresholds; instead, discomfort is used at a low pressure, and the other labels are applied inconsistently. This pattern was verified by testing the difference in linear trend for the two groups across the seven descriptors (Wald $\chi^{2}=8.1, \mathrm{p}<0.01$ ).

STIMULUS RESPONSE CURVES FOR THE ASCENDING SERIES

The sensory intensity and unpleasantness ratings made during the ascending pressure series (stimulus response (S-R) curves) were analysed by comparing the IBS and control groups across the pressure steps between 10 and $45 \mathrm{~mm} \mathrm{Hg}$. Each analysis used a repeated measures analysis of variance, which compared the two groups on the ratings of the eight stimuli. ${ }^{22}$ No significant overall group differences in ratings were found, nor were there differences between the groups in the slopes of the stimulus response curves for either verbal descriptor scale. Figure 4 shows these S-R curves. Overall, the affective ratings show a flatter S-R curve compared with the sensory ratings. This indicates that subjects were using the descriptors as a guide to scale the stimuli, and were making some differentiation between sensory and affective qualities of the visceral stimuli. All subjects tolerated up to $45 \mathrm{~mm} \mathrm{Hg}$. A small number of subjects in the control group (14\%) and $29 \%$ of the patients with IBS reached their tolerance at $45 \mathrm{~mm} \mathrm{Hg}$, and higher pressures were not given ( $\chi^{2}$ for group difference non-significant). A significantly greater percentage of patients with IBS (72\%) compared with controls (29\%) would not tolerate pressures above $50 \mathrm{~mm} \mathrm{Hg}$ $(\mathrm{p}<0.01)$. A separate analysis of the $S-R$ curves

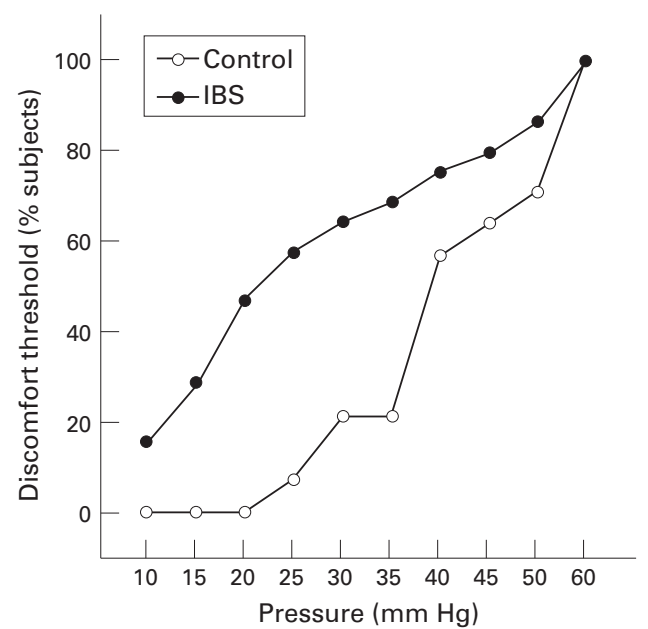

Figure 2: Cumulative percentage of subjects reaching discomfort threshold during the ascending series by balloon pressure for the IBS and control groups. 


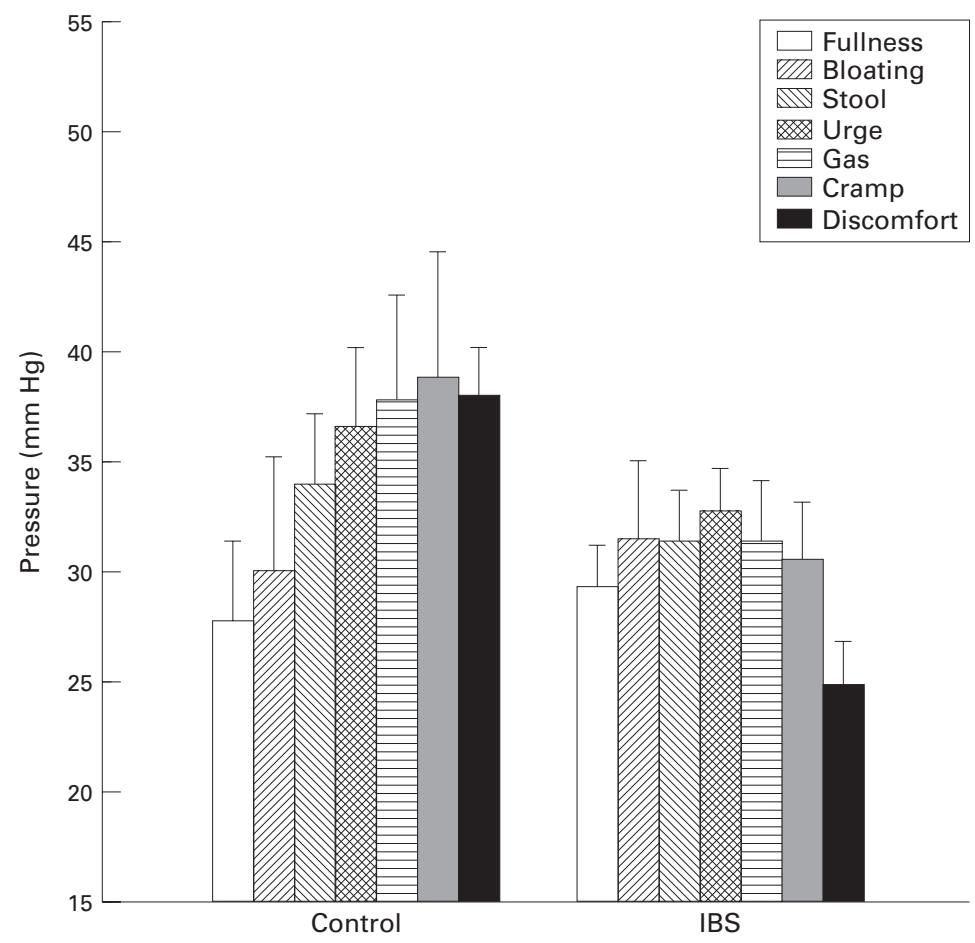

Figure 3: Average thresholds for first use of the various visceral sensations for the control and IBS groups from the ascending series. Error bars are SEM.

was performed for subjects which tolerated the entire pressure range of $10-60 \mathrm{~mm} \mathrm{Hg}$. Overall, subjects who tolerated $60 \mathrm{~mm} \mathrm{Hg}$ had lower affective ratings of the stimuli up to $45 \mathrm{~mm} \mathrm{Hg}$ than subjects who stopped the procedure at lower pressures (for affective scale, $\mathrm{F}=15.66, \mathrm{p}<0.01$; for sensory, $\mathrm{F}=3.85$, $\mathrm{p}=0.06$ ). However, there were no differences between the IBS and control groups in the S-R curves for the subjects who completed the task. The sensory data from the ascending series therefore indicate lower discomfort thresholds and lower tolerance for the $50 \mathrm{~mm} \mathrm{Hg}$ stimulus for patients with IBS compared with controls, but no differences in S-R curves for stimuli up to $45 \mathrm{~mm} \mathrm{Hg}$.

WALL TENSION DURING THE ASCENDING SERIES Wall tension curves were also analysed in an analogous fashion to the S-R curves. There were overall group differences in the wall tension curves for both wall tension at target pressure and maximum volume (for target pressure, $\mathrm{F}=4.31, \mathrm{p}<0.05$; for maximum volume, $\mathrm{F}=9.61, \mathrm{p}<0.01)$. In both cases the IBS group showed greater compliance (decreased wall tension) across the pressure steps. Analysis of covariance was used to evaluate whether differences in wall tension might account for the lack of group differences in S-R curves. The wall tension at each pressure step was entered as a covariate in a separate analysis of the $S-R$ curves. The results did not change; there were no differences between the IBS and control groups in the sensory or affective $S-R$ curves.

DISCOMFORT THRESHOLDS FROM THE STIMULUS TRACKING TASK

There were no significant group differences in tracking thresholds or tracking reliability (de-
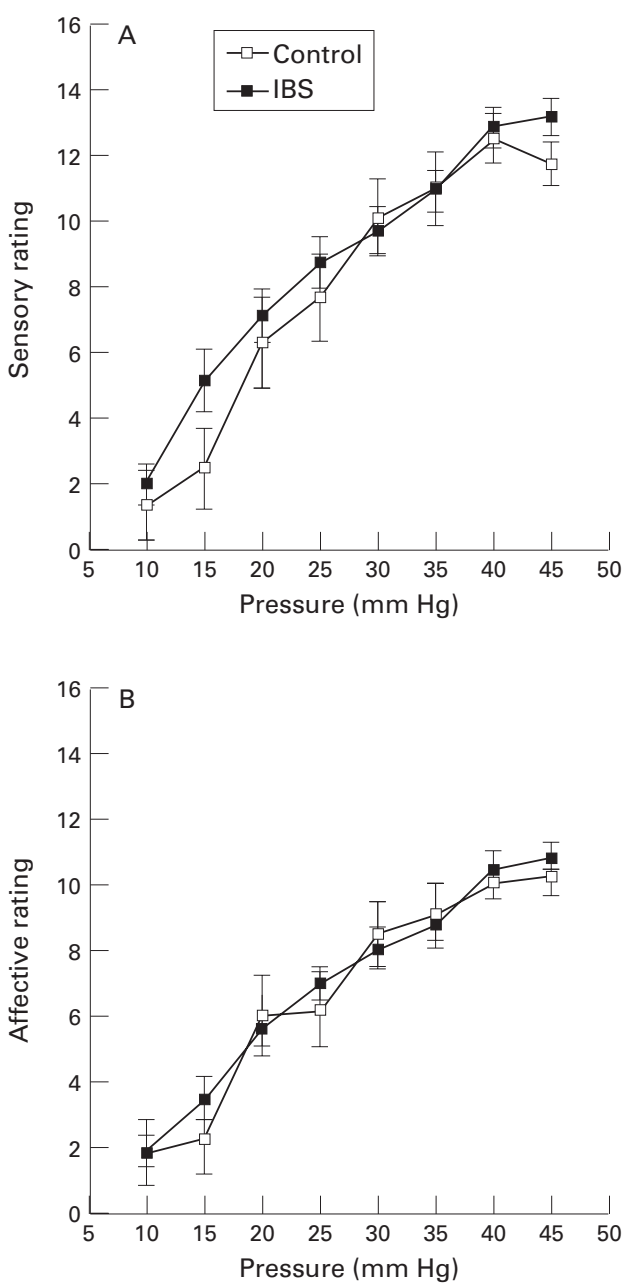

Figure 4: $\quad S$-R curves for the sensory $(A)$ and affective (B) verbal descriptor scales during the ascending series of rectal distensions. Error bars are SEM.

termined from the standard deviation of responses for the last six tracking trials). The average tracking threshold for the patients with IBS was 27.7 (1.5) $\mathrm{mm} \mathrm{Hg}$, and for the controls was 30.4 (1.9) $\mathrm{mm} \mathrm{Hg}$. Optimal sensitivity of a tracking protocol occurs when the pressures vary above and below the discomfort threshold. To check for bias in the results, thresholds determined by averaging only consecutive pressure pairs which were associated with a transition of ratings from below to above, or above to below, discomfort were also examined. Again, no group differences were found. The lower bound of the $95 \%$ confidence interval for the discomfort threshold of control subjects was $25.2 \mathrm{~mm} \mathrm{Hg}$. Forty seven per cent of patients with IBS and $16 \%$ of controls fell below this value ( $\chi^{2}$ for group differences was non-significant).

FACTOR ANALYSIS OF PSYCHOPHYSICAL VARIABLES Factor analysis is an analytical tool for summarising multiple measurements into a small number of reliable dimensions. A principal components factor analysis with Varimax rotation $^{23}$ was run in order to explore the relationship among the main psychophysical variables for the patients with IBS: discomfort 
threshold and tolerance during the ascending series, discomfort threshold during the tracking task, and sensory and affective scaling of a generally uncomfortable stimulus $(45 \mathrm{~mm} \mathrm{Hg})$. Two factors were identified (eigenvalues $>1.0$ ) which together accounted for $71 \%$ of the total variance. Table 1 shows rotated factor loadings from this analysis. Factor 1 had high loadings from the tracking thresholds as well as the sensory and affective ratings during the ascending series. Based on these loadings this factor has been labelled visceral sensitivity. Factor 2 had the highest loadings from the tolerance and discomfort threshold measures during the ascending series. This factor appears to reflect tolerance and response bias towards reporting discomfort and therefore might be associated with visceral hypervigilance. ${ }^{24}$

HYPERSENSITIVE VERSUS NORMOSENSITIVE PATIENTS WITH IBS

It has previously been suggested that patients who have normal perception thresholds to rectal distension and those who are hypersensitive represent different patient subpopulations, with distinct pathophysiologies. ${ }^{35}$ In previous studies hypersensitivity has been defined as a discomfort threshold below the 95\% confidence interval of a normal control group. ${ }^{325}$ In order to examine the data from the current study in a manner consistent with this dichotomous grouping of patients, we split our IBS sample into patients whose tracking discomfort threshold fell below the $95 \%$ confidence interval of the control subjects, and those whose thresholds were within the normal range. We then compared these two groups in terms of other perceptual responses, clinical and psychological parameters.

Both the sensory intensity and affective S-R curves for $10-45 \mathrm{~mm} \mathrm{Hg}$ were significantly different between the hypersensitive and normosensitive patients with IBS (for sensory, $\mathrm{F}=3.95, \mathrm{p}<0.05$; for affective, $\mathrm{F}=4.18$, $\mathrm{p}<0.05)$. Figure 5 shows these $S-R$ curves. There were no interactions between pressure level and group indicating that the curves are different across the entire pressure range. However, the hypersensitive and normosensitive patients did not differ on the first use of discomfort as assessed during the ascending pressure series (27.4 (4.1) $\mathrm{mm} \mathrm{Hg}$ for hypersensitive and $31.2(3.1) \mathrm{mm} \mathrm{Hg}$ for normosensitive). Thus, despite being selected for differences on tracking discomfort threshold, the hypersensitive and normosensitive subjects with IBS did not differ on the discomfort threshold during the ascending series. Both the hypersensitive and normosensitive groups have

TABLE 1 Rotated factor loadings of psychophysical variables

\begin{tabular}{lll}
\hline & $\begin{array}{l}\text { Factor 1 } \\
\text { Sensitivity }\end{array}$ & $\begin{array}{l}\text { Factor 2 } \\
\text { Hypervigilance }\end{array}$ \\
\hline Ascending-tolerance & NA & 0.85 \\
Ascending-threshold & NA & 0.70 \\
Sensory rating (45 mm Hg) & 0.90 & NA \\
Affective rating (45 mm Hg) & 0.88 & NA \\
Tracking-threshold & -0.78 & NA \\
\hline
\end{tabular}

Note: only factor loadings over 0.30 are shown.
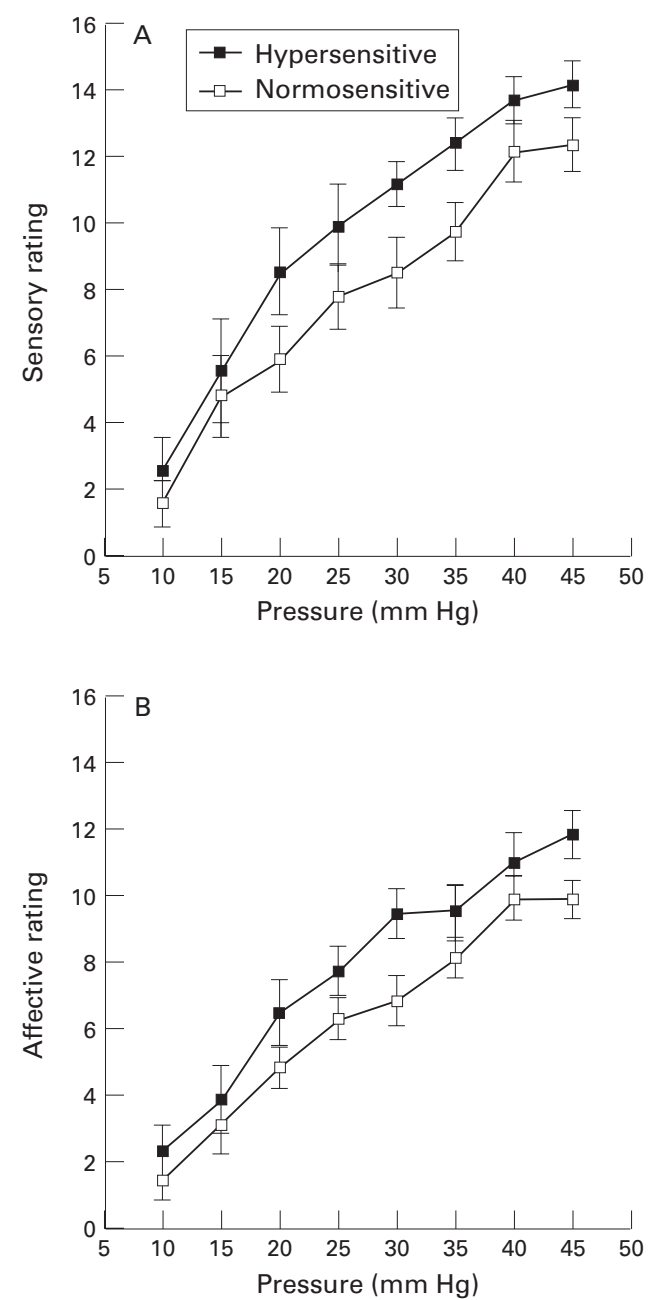

Figure 5: $S-R$ curves for the sensory $(A)$ and affective (B) verbal descriptor scales during the ascending series of rectal distensions for hypersensitive and normosensitive patients with IBS. Error bars are SEM.

lower ascending series discomfort thresholds compared with the control group $(\mathrm{p}<0.01)$. The hypersensitive and normosensitive patients with IBS also did not differ on any of the psychological symptom scales or sex.

SENSORY AND UNPLEASANTNESS RATINGS OF VISCERAL SENSATION DESCRIPTORS

The sensory testing results reported above indicate that IBS and control subjects differ in their labelling of visceral sensations evoked by rectal balloon distension. In order to examine directly differences in how visceral sensations are viewed between these groups, we asked separate samples of IBS $(n=17)$ and control $(n=17)$ subjects to rate the sensory intensity and unpleasantness of each of the visceral sensation descriptors (no sensation, abdominal cramps, bloating, fullness, gas, stool, urgency, discomfort). The visceral descriptors were presented twice in random order along with either a sensory or unpleasantness scale. ${ }^{12}$ Patients with IBS rated the descriptor "discomfort" significantly higher on unpleasantness compared with the controls $(7.5(0.64)$ for controls; 10.3 (1.1) for IBS; $\mathrm{p}<0.05)$. The closest unpleasantness cues along the visual 
analogue scale corresponding to the control mean rating was "annoying" and to the IBS mean rating was "distressing". Patients with IBS also rated the visceral descriptor "stool" significantly higher than controls on the intensity scale (7.1 (1.1) for controls; 11.2 (1.2) for IBS; $\mathrm{p}<0.05)$. The closest intensity cues for these ratings were "mild" and "moderate" respectively.

\section{Discussion}

This study applied three psychophysical techniques in order to assess perceptual responses to rectal balloon distension in patients with IBS and healthy controls. Two potentially distinct perceptual alterations have been identified which may be related to different neurophysiological mechanisms. Firstly, $47 \%$ of patients with IBS show rectal hypersensitivity in the form of lowered discomfort thresholds during tracking and a left shift of the stimulus response curve. Secondly, regardless of the presence of hypersensitivity, patients with IBS used the descriptor "discomfort" at a significantly lower distension pressure, and showed less tolerance for distension during an ascending series of stimuli compared with controls. The separation of the various psychophysical variables into these two categories is reinforced by factor analysis which found two factors: tracking discomfort thresholds and S-R curve ratings; and ascending discomfort thresholds and tolerance. IBS and control subjects also differed in their unpleasantness ratings of common visceral sensations, with the IBS subjects rating "discomfort" as more unpleasant than controls. These findings, together with results from previous sensory studies in IBS and other functional gastrointestinal syndromes, suggest a model of overlapping sensory and psychological mechanisms in these disorders.

HYPERSENSITIVITY TO VISCERAL STIMULI

No overall group difference was found between patients with IBS and controls in average discomfort threshold from the tracking task. However, almost half of the patients with IBS had clear evidence of rectal hypersensitivity with tracking discomfort thresholds below the 95\% confidence interval of that for the controls. Despite the fact that clinical practice usually involves management of moderate to intense discomfort, most research has focused on threshold level visceral sensations. It is therefore of particular importance that the hypersensitive patients with IBS identified by tracking threshold, also show a significant left shift of their sensory and unpleasantness stimulus response curves across all stimulus intensities. The validity of using tracking thresholds as an objective measure of sensitivity (or hypersensitivity) is reinforced by the association of this threshold with the S-R curves of sensory and affective intensity ratings.

The reason why only a subgroup of patients with IBS exhibit rectal hypersensitivity under baseline conditions is open to question. Rectal sensitivity was not correlated with psychological symptom scores, sex, or age. Hypersensitivity may not be a constant feature of patients with IBS over time, but may be unmasked dur- ing periods of internal or external stress. We have recently reported that rectal tracking thresholds for discomfort in patients with IBS are reliably modified from normosensitive to hypersensitive following prolonged high pressure stimulation of the sigmoid colon, and that this propensity for developing hypersensitivity (sensitisation) is specific for patients with IBS. ${ }^{25}$ Thus, the increased and prolonged sigmoid contractile response to food intake ${ }^{26}$ and emotional stress ${ }^{27}$ reported in patients with IBS may be responsible for transiently inducing rectal hypersensitivity in otherwise normosensitive patients with IBS. The significance of rectal hypersensitivity in a small subgroup of healthy controls is unclear given the sample size of this group.

HYPERVIGILANCE TO VISCERAL STIMULI

We confirmed our previous observation ${ }^{3}$ that the descriptor discomfort was used at a significantly lower distension pressure in patients with IBS compared with controls during an ascending series of rectal stimuli. In contrast to the preferred use of "discomfort" in the IBS group, there were no differences between the groups in the average thresholds for use of the other qualitative descriptors (fullness, bloating, stool, urgency, gas, and cramps), nor a group difference in the total number of descriptors used during the task. These findings, coupled with a lower tolerance for pressures above $50 \mathrm{~mm} \mathrm{Hg}$ during the ascending series, suggest an aberrant behavioural response related to hypervigilance for rectal stimuli in IBS. The cognitive bias implied by these results extends to evaluations of the visceral sensation descriptors themselves. When compared with other visceral descriptors, the relatively non-specific descriptor "discomfort" was judged most unpleasant by the patients with IBS, and was rated both absolutely and relatively lower by controls. Patients with IBS, therefore, both view visceral discomfort as more unpleasant, and experience it at a lower stimulus level compared with controls. Selective cognitive bias in IBS for negative words (unrelated to visceral sensations) has been previously demonstrated using a recall task. ${ }^{10}$ Similar to the present results, the bias could not be accounted for by presence of depressed mood. In another recent study of patients with IBS, organic gastrointestinal disease, and depression, it was also shown that neither depression nor the experience of gastrointestinal symptoms could account for the abnormal illness attitudes associated with IBS. ${ }^{11}$

In contrast to the unpredictable nature of each stimulus during the tracking task, the predictable increase in stimulus magnitude during the ascending series allows for bias in subject judgements. Non-sensory cues such as a biased recall for negative words, ${ }^{10}$ anticipation of a higher level stimulus, and fear of increasing discomfort, may all play a role in patients with IBS' tendency to label visceral sensations in aversive terms. Such cues are unlikely to influence ratings significantly in the tracking task. This tendency to select an affective descriptor (discomfort) early in the ascending series, may 
have also resulted in the patients with IBS' less consistent use of the other descriptors as shown in the analysis of threshold order in both this study and our previous study. ${ }^{28} \mathrm{~A}$ similar increased tendency to use non-sensory cues to rate expected visceral distension stimuli has been reported in patients with other functional gastrointestinal disorders. Richter and Bradley, using a sensory decision theory analysis, have shown that patients with non-cardiac chest pain have lowered discomfort thresholds which are associated with response bias and not sensory discrimination. ${ }^{29}$ More recently, Mertz et al found that patients with functional dyspepsia have a greater tendency to use the descriptor "pain" during gastric sham distension. ${ }^{30}$

The concept of greater vigilance in patients with IBS towards expected visceral discomfort is further supported by recent studies using positron emission tomography to identify regional brain responses associated with delivered and anticipated rectal pain. Patients with IBS as a group showed aberrant activation of the left dorsolateral prefrontal cortex in anticipation of rectal pain without any stimulus being delivered ${ }^{31}$; this brain region is involved in the attachment of significance to sensory events and plays a part in the development of conditioned fear responses. ${ }^{32}$

The aim of the present study was to characterise perceptual responses in patients with IBS whose symptoms were severe enough that they sought continued health care and presented at a specialised programme for anorectal disorders. The findings may not be applicable to the large group of individuals with IBS symptoms who have not sought health care and who show distinctly different psychological profiles from IBS clinic patients. ${ }^{9}$ In our previous studies ${ }^{3}{ }^{17}$ we have not found that self rated severity of symptoms in health care seeking patients is correlated with alterations in perceptual responses.

In summary, our findings provide evidence for the presence of two distinct perceptual alterations in patients with IBS: hypersensitivity to rectal distension; and hypervigilance (or change in response criteria) towards labelling a wide range of visceral stimuli in negatively affective terms. One may speculate about the possible inter-relationship between these perceptual alterations in patients with IBS. The often life long experience of recurring abdominal pain and discomfort may be related to intermittent visceral hypersensitivity, which can be detected in a subset of patients with IBS at a given time in a laboratory situation, and which may be unmasked by intestinal irritation such as repetitive high amplitude contractions. ${ }^{25}$ This chronic experience of visceral pain and discomfort may condition some patients to label any sensations arising from the lower gastrointestinal tract in negatively charged terms leading to a positive response bias in reporting discomfort.

This work was supported by National Institute of Diabetes and Digestive and Kidney Diseases Grant DK48351 and the Department of Veterans Affairs Medical Research Program.

1 Kellow JE, Eckersley CM, Jones MP. Enhanced perception of physiological intestinal motility in the irritable bowel syndrome. Gastroenterology 1991; 101: 1621-7.
2 Bradette M, Delvaux M, Staumont G, Fioramonti J, Bueno Frexinos J. Evaluation of colonic sensory thresholds in L, Frexinos J. Evaluation of colonic sensory thresholds in
IBS patients using a barostat. Dig Dis Sci 1994; 39: 449-57. 3 Mertz H, Naliboff B, Munakata J, Niazi N, Mayer E. Altered rectal perception is a biological marker of patients with the irritable bowel syndrome. Gastroenterology 1995; 109: 40-52.

4 Whitehead WE, Holtkotter B, Enck P, Hoelzl R, Holmes $\mathrm{KD}$, Anthony J, et al. Tolerance for rectosigmoid distension in irritable bowel syndrome. Gastroenterology 1990; 98: 1187-92.

5 Prior A, Sorial E, Sun W-M, Read NW. Irritable bowel syndrome: differences between patients who show rectal sensitivity and those who do not. Eur $\mathcal{F}$ Gastroenterol Hepatol 1993; 5: 343-9.

6 Lipkin M, Sleisenger MH. Studies of visceral pain: measurements of stimulus intensity and duration associated with the onset of pain in esophagus, ileum and colon. 7 Clin Invest 1957; 37: 28-34.

7 Gracely, RH. Methods of testing pain mechanisms in normal man. In: Wall PD, Melzack, R, eds. Textbook of pain. New York: Churchill Livingstone, 1989: 257-68.

8 Naliboff B, Mayer EA. Commentary: sensational developments in the irritable bowel. Gut 1996; 39: 770-1.

9 Drossman DA, McKee DC, Sandler RS, Mitchell CM, Cramer EM, Lowman BC, et al. Psychosocial factors in the irritable bowel syndrome. A multivariate study of patients and nonpatients with irritable bowel syndrome. Gastroenterology 1988; 95: 701-8.

10 Gomborone JE, Dewsnap PA, Libby GW, Farthing MJ. Selective affective biasing in recognition memory in the irritable bowel syndrome. Gut 1993; 34: 1230-3.

11 Gomborone J, Dewsnap P, Libby G, Farthing M. Abnormal illness attitudes in patients with irritable bowel syndrome. $\mathcal{F}$ Psychosom Res 1996; 39: 227-30.

12 Gracely RH, McGrath P, Dubner R. Ratio scales of sensory and affective verbal pain descriptors. Pain 1978; 5: 5-18.

13 Naliboff B, Munakata J, Gracely R, Fullerton S, Kodner A, Mayer EA. IBS patients with low rectal discomfort thresholds show normal stimulus response functions for rectal sensations [abstract]. Gastroenterology 1995; 108: A656.

14 Thompson WG, Creed F, Drossman DA, Heaton KW, Mazzacca G. Functional bowel disease and functional abdominal pain. Gastroenterology International 1992; 5: 75-91.

15 Manning AP, Thompson WD, Heaton KW. Towards positive diagnosis in the irritable bowel syndrome. $B M \mathcal{F}$ 1978; 2: 653-4.

16 Derogatis LR. SCL-90R. Administration, scoring and procedures manual -II. Baltimore, MD: Baltimore Clinical Psychometric Research, 1983.

17 Lembo T, Munakata J, Mertz H, Niazi N, Kodner A, Nikas $\mathrm{V}$, et al. Evidence for the hypersensitivity of lumbar splanchnic afferents in irritable bowel syndrome. Gastroenterology 1994; 107: 1686-96.

18 Mertz H, Walsh JH, Sytnik B, Mayer EA. The effect of octreotide on human gastric compliance and sensory perception. Neurogastroenterol Motil 1995; 7: 175-85.

19 Whitehead WE, Crowell MD, Shone D, Davidoff A, Cheskin LC, Schuster MM. Sensitivity to rectal distension: validation of a measurement system [abstract]. Gastroenterology 1993; 104: A600.

20 Cornsweet TN. The staircase-method in psychophysics. Am 7 Psychol 1996; 75: 485-91.

21 Silverman DH, Munakata JA, Ennes H, Mandelkern MA, Silverman DH, Munakata JA, Ennes H, Mandelkern MA,
Hoh CK, Mayer EA. Regional cerebral activity in normal Hoh CK, Mayer EA. Regional cerebral activity in normal and pathological percept

22 Kirk RE. Experimental design: procedures for the behavioral sciences. Monterey, CA: Brooks/Cole Publishing Co., 1982.

23 Norusis MJ. SPSS/PC+ Advanced statistics V 2.0, Chicago, IL: SPSS Inc., 1988.

24 Chapman, CR. Pain: the perception of noxious events. In: Sternbach RA, ed. The psychology of pain. New York: Raven Press, 1978: 169-202.

25 Munakata J, Naliboff B, Harraf F, Kodner A, Lembo T, Chang $\mathrm{L}$, et al. Repetitive sigmoid stimulation induces rectal hyperalgesia in patients with irritable syndrome. Gastroenterology 1997; 112: 55-63.

26 Bazzocchi G, Ellis J, Villanueva-Meyer J, Reddy SN, Mena I, Snape WJ Jr. Effect of eating on colonic motility and transit in patients with functional diarrhea. Gastroenterology transit in patients with

27 Welgan P, Meshkinpour H, Hoehler F. The effect of stress on colon motor and electrical activity in irritable bowel syndrome. Psychosom Med 1985; 47: 139-49.

28 Harraf F, Fass R, Diehl D, Niazi N, Kodner A, Mertz H, et al. Symptom based subtypes of constipation are correlated with rectal sensitivity [abstract]. Gastroenterology 1996; 110: A675.

29 Richter JE, Bradley LA. The irritable esophagus. In: Mayer EA, Raybould HE, eds. Basic and clinical aspects of chronic abdominal pain. Amsterdam: Elsevier, 1993.

30 Mertz H, Fass R, Hirsh T, Yan-Go F, Mayer EA. Amitryptiline for functional dyspepsia: effect on symptoms, gastric sensitivity and sleep [abstract]. Gastroenterology 1995; 108: A649.

31 Silverman DHS, Munakata J, Hoh CK, Mandelkern M, Phelps ME, Blahd W, et al. Regional cerebral activity in normal and pathologic perception of visceral pain: an normal and pathologic perception of visceral pain: an O-15-water PET study of healthy subjects and
[abstract]. Gastroenterology 1996; 110: A759.

32 Devinsky O, Morrell MJ, Vogt BA. Contributions of anterior cingulate cortex to behaviour. Brain 1995; 118: 279-306. 